\title{
Dynamic expression of the cell adhesion molecule fasciclin I during
} embryonic development in Drosophila

\author{
LINDA MCALLISTER*, COREY S. GOODMAN \\ Howard Hughes Medical Institute, Department of Molecular and Cell Btology, University of California, Berkeley, Berkeley, CA 94720, USA
}

and KAI ZINN

Division of Biology 216-76, California Institute of Technology, Pasadena, CA 91125, USA

* Present address Department of Internal Medicine, University of California, San Francisco, CA 94143

\section{Summary}

A number of different cell surface glycoproteins expressed in the central nervous system (CNS) have been identified in insects and shown to mediate cell adhesion in tissue culture systems. The fasciclin I protein is expressed on a subset of CNS axon pathways in both grasshopper and Drosophila. It consists of four homologous 150-amino acid domains which are unrelated to other sequences in the current databases, and is tethered to the cell surface by a glycosyl-phosphatidylinositol linkage. In this paper we examine in detail the expression of fasciclin I mRNA and protein during Drosophila embryonic development. We find that fasciclin $I$ is expressed in several distinct patterns at different stages of development. In blastoderm embryos it is briefly localized in a graded pattern. During the germ band extended period its expression evolves through two distinct phases. Fasciclin I mRNA and protein are initially localized in a 14-stripe pattern which corresponds to segmentally repeated patches of neuroepi- thelial cells and neuroblasts. Expression then becomes confined to CNS and peripheral sensory (PNS) neurons. Fasciclin I is expressed on all PNS neurons, and this expression is stably maintained for several hours. In the CNS, fasciclin I is initially expressed on all commissural axons, but then becomes restricted to specific axon bundles. The early commissural expression pattern is not observed in grasshopper embryos, but the later bundle-specific pattern is very similar to that seen in grasshopper. The existence of an initial phase of expression on all commissural bundles helps to explain the loss-of-commissures phenotype of embryos lacking expression of both fasciclin $I$ and of the $D$ - $a b l$ tyrosine kinase. Fasciclin $I$ is also expressed in several nonneural tissues in the embryo.

Key words: adhesion molecule, Drosophila development, axon guidance, neural development.

\section{Introduction}

Fasciclin $\mathrm{I}$ is a $72 \times 10^{3} M_{\mathrm{r}}$ glycoprotein that was originally identified by a monoclonal antibody (mAb) screen for molecules expressed on subsets of CNS axons in embryos of the grasshopper Schistocerca americana (Bastiani et al., 1987). Cell ablation experiments in this organism had provided evidence that CNS growth cones use specific axon bundles as guidance cues (reviewed by Goodman et al., 1984), and the screen was designed to identify surface molecules whose expression patterns correlated with a potential role in guidance. Two other molecules, fasciclin II (in grasshopper) and fasciclin III (in Drosophila melanogaster), were also identified in these types of screens (Bastiani et al., 1987; Patel et al., 1987; Harrelson and Goodman, 1988; Snow et al., 1988). The cloned sequences for fasciclin I and II were used to isolate homologs in Drosophila (Zinn et al., 1988; Grenningloh et al., 1990, 1991).

The sequence of fasciclin $\Pi$ is closely related to that of the vertebrate neural adhesion molecule N-CAM (Harrelson and Goodman, 1988). All three fasciclins are capable of mediating cell adhesion in tissue culture systems (Snow et al., 1989; Elkins et al., 1990b; Grenningloh et al., 1990).

To gain insights into the functions of these molecules during neural development, mutations in the Drosophila genes encoding the three fasciclins have been isolated (Elkins et al., 1990a; Grenningloh et al., 1991; T. Elkins and C. S. Goodman, unpublished results). Apparent null mutations in the fasciclin $I$ (fasI) and fasciclin III (fasIII) genes are homozygous viable, while a null fasciclin II (fasII) mutation causes lethality when 
homozygous. FasII mutations prevent formation of a specific longitudinal pathway that expresses the protein (Grenningloh et al., 1991). The other mutations do not produce alterations in the embryonic CNS that can be visualized with currently available antibody reagents. Defects in individual pathways are usually very difficult to detect, however, because few axon-specific antibodies exist. For fasciclin II, it was possible to visualize the affected longitudinal pathway in fas $I I$ mutants with the $22 \mathrm{C} 10 \mathrm{mAb}$, which labels a restricted set of axons in stage 12 and 13 embryos (Grennıngloh et al., 1991).

Analyses of antibody-stained embryonic CNS preparations from fas $I$ and fas $I I I$ mutants do show that many of the axons that express these proteins follow normal pathways in the mutant embryos, suggesting that if they are involved in axon guidance their functions can be substituted for by other molecules (Elkins et al., 1990a; T. Elkins, R. Jacobs, and C.S.G., unpublished results). This apparent functional redundancy correlates with observations made on vertebrate cell adhesion molecules in tissue culture systems, where it has been found that antıbodies against several different molecules are required to block axon fasciculation or neurite outgrowth (Tomaselli et al., 1986; Chang et al., 1987; Neugebauer et al., 1988).

In the case of fasciclin I, a requirement for this molecule in embryonic neural development can be uncovered by constructing a double mutant stock lacking expression of both fasciclin I and of the Drosophila homolog of the $a b l$ tyrosine kinase, which is expressed on CNS axons (Gertler et al., 1989). In fasI/abl mutant embryos, the commissural axon pathways are often completely absent. The mutant combination can also be shown to alter pathfinding by identified neurons. The fasciclin I-positive RP1 motoneurons normally extend growth cones across the midline and exit the CNS in the contralateral intersegmental nerve (ISN) pathway, but in mutant embryos their axons usually fail to cross over and instead grow out in the ipsilateral ISN (Elkins et al., 1990a). The $a b l$ mutation alone does not cause embryonic lethality, and no gross alterations in the CNS are observed in $a b l$ mutant embryos (Henkemeyer et al., 1987; Gertler et al., 1989).

The synergistic interaction between the fasI and $a b l$ mutations suggests that fasciclın I does not simply mediate adhesion, but participates in a signal transduction pathway involved in growth cone extension or guidance. This pathway may function in parallel with a pathway involving $a b l$, so that elimination of one pathway does not cause severe defects, but removing both pathways prevents correct formation of the CNS axon array.

The sequence of the fasciclin I protein does not clearly define its function, as it is not related to other sequences in the current databases. It consists of four homologous 150 amino acid domains, and is linked to the external cell surface by a glycosyl-phosphatidylinositol (GPI) linkage (Zinn et al., 1988; Hortsch and Goodman, 1990). Alternative splicing of two 6-9 bp micro-exons located between the coding regions for the second and third domains produces mRNAs encoding three different isoforms of the protein in each species (McAllister et al., 1992). In the present study, we have examined in detail the pattern of expression of fasciclin I mRNA and protein during development of the Drosophila embryo. These results help to explain the origin of the fasI/abl phenotype, and may provide insights into the possible roles of this molecule in embryonic development.

\section{Materials and methods}

\section{Immunocytochemistry with anti-fasciclin I mAbs}

Immunocytochemistry on whole-mount embryos was performed according to Patel et al (1987, 1989). Ascites preparations (1:500) or cell supernatants $(1: 2)$ of the antifasciclın I monoclonal antıbody 6D8 (Hortsch and Goodman, 1990) were used as the primary antibody Photographs were taken using Nomarski optics on either a Zeıss Axiophot or a Nikon compound microscope.

\section{Whole mount in situ hybridization with fasciclin I probes}

In situ hybridization to whole mount embryos was performed essentially according to the protocol of Tautz and Pfeifle (1989). Non-radioactive probes were made by incorporating digoxigenın-dUTP (Boehringer Mannheum) either by prımıng with random hexamers or by synthesizing anti-sense singlestranded DNA probe using a single primer PCR reaction. Digoxigenin was detected using a monoclonal antıbody against digoxigenın coupled to alkalıne phosphatase. The alkaline phosphatase reaction was carried out using NBT and $\mathrm{X}$-phosphate as supplıed in the Boehringer Mannheim Genıus detection kıt, or with a Vector Laboratories ABC kit. Photographs were taken as for antibody stainıng.

\section{Results}

Dynamıc expression of fasciclin I $m R N A$ and protein during blastoderm and gastrula stages

We examıned the expression of fasciclin I RNA during development using the digoxigenin whole-mount in situ hybridization method of Tautz and Pferfle (1989). There are two major fasciclin I transcripts, of 3.0 and $5.0 \mathrm{~kb}$, which differ only in their $3^{\prime}$ untranslated regions. We used both a coding region probe and a probe specific to the large RNA. Identical RNA expression patterns were observed with the two probes (data not shown). Fasciclin I protein expression was visualized using horseradish peroxidase (HRP) immunohistochemistry with a mAb raised against a bacterially expressed fasciclın I/protein A fusion (Hortsch and Goodman, 1990).

Fasciclin I mRNA is maternally deposited in the egg, and the message is initially uniformly distributed. At about $1 \mathrm{~h}$ after egg layıng (AEL) the nuclei, which have undergone 7 synchronous divisions in the central region of the egg, begin to migrate out to the penphery. The fasciclin I RNA hybridization signal becomes localized with the nuclei, so that it forms a broad annulus (data not shown). After the nucles reach the periphery they 
divide three more times, and then cellularization begins. Just before cellularization, fasciclin I RNA is observed in a ring at the level of the nuclei (Fig. 1A).

Fasciclin I protein is initially uniformly distributed on the surface of the egg, and it first appears when the outlines of the forming cell membranes become evident. During the cellularization process there is a period of about $10-15 \mathrm{~min}$ in which fasciclin I protein is expressed at a low level in the anterior third of the egg and at a higher level in the posterior two thirds. The highest levels of protein observed at this time are on the membrane at the extreme posterior end of the egg, adjoining the pole cells (Fig. 1B). Pole cells are the germ line precursors, and segregate away from the rest of the embryo very early in development, at the time of the 9 th cleavage division. The pole cells themselves do not express the protein at this time. After this stage, fasciclin I protein again becomes uniformly distributed, and at the beginning of gastrulation $(2: 50 \mathrm{~h} \mathrm{AEL}$, stage 6 of Campos-Ortega and Hartenstein, 1985) it is expressed at a high level on all cell membranes. During gastrulation (2:50-3:20 h AEL), fasciclin I mRNA is apparently uniformly distributed (data not shown). The protein, however, is expressed at higher levels on the invaginating cells of the ventral and cephalic furrows (Fig. 1C).

Immunohistochemical analysis of the progeny of crosses in which there is no maternal fasciclin I expression (fasI $I^{T E} /$ fas $^{T E}$ females $\times+/+$ males), or in which $1 / 2$ of the progeny lack zygotic fasciclin I expression $\left(+/\right.$ fasI $^{T E}$ females $\times$ fasI ${ }^{T E} /$ fasI $^{T E}$ males $)$ indicates that the transition between maternal and zygotic expression takes place at about the tıme of gastrulation (data not shown).

\section{Two phases of fasciclin 1 expression in germ band extended embryos}

Immediately following gastrulation, the fast phase of germ band elongation begins. The germ band refers to the segmented region of the embryo, which gives rise to three gnathal, three thoracic, and at least nine abdominal segments. During germ band elongation the segmental primordia fold over the posterior end of the embryo and extend forward as far as $75 \%$ egg-length (EL; measured from the posterior end). The embryo remains in the germ band extended stage for several hours (3:40-7:20 hours AEL, stages 9-11). During this time neuroblasts delaminate from the ectoderm and divide asymmetrically to produce a chain of ganglion mother cells (GMCs). Each GMC then divides once, giving rise to two neurons. This process eventually generates approximately 250 neurons per hemisegment, and these will form the segmental ganglia of the CNS.

In the 4-5 h old embryo fasciclin I RNA is localized in 14 stripes, which correspond to segmentally repeated bands of neuroepithelial cells and delaminating neuroblasts (Fig. 1D, stage 9). The protein has a similar distribution, although it appears slightly later and seems to be expressed at lower levels in the epithelial layer (Fig. 1E, stage 10). These stripes of neuroblasts and neuroepithelial cells are localized to the posterior compartment of the segment, or the anterior region of the parasegment (Martinez-Arias and Lawrence, 1985; data not shown). This was determined by double staining with anti-fasciclin I mAb and antibodies against $\beta$-galactosidase in a transgenic line containing a $\beta$-galactosidase gene under the control of the promoter of the fushi tarazu gene, which is expressed in 7 stripes at the anterior border of alternating parasegments (Hafen et al., 1984).

In later germ band extended embryos the hybridization signal moves inward into the embryo and becomes a single longitudinal stripe running the entıre length of the segmented region (Fig. 1F, stage 11). Immunocytochemistry with the mAb shows that the protein has a similar distribution (data not shown). This stripe corresponds to the neuronal layer, in which many cells express fasciclin I. These cells are localized in both compartments of the segment, so a banded pattern is no longer observed. We do not know if the early neurons expressing fasciclin I are primarily derived from the fasciclin I-positive neuroblasts.

\section{Stable fasciclin I expression in the peripheral nervous system}

Each thoracic and abdominal segment of the embryo contains five classes of peripheral sensory neurons localized in three clusters: the ventral, lateral, and dorsal clusters (Campos-Ortega and Hartenstein, 1985; Ghysen et al., 1986). The external sensory (es) neuron and chordotonal (ch) neuron classes both have single dendrites, and there are three classes of neurons with multiple dendntes (md neurons). The precursors of these PNS neurons undergo their final mitoses at about 6:30 h AEL (Bodmer et al., 1989). The dorsal cluster appears first, followed closely by the lateral and ventral clusters. These cells then extend axons into the CNS, and the mature pattern of PNS neurons appears by about $11 \mathrm{~h}$ AEL.

At the beginning of germ band retraction (about $7 \mathrm{~h}$ AEL) small, segmentally repeated clusters of fasciclin I RNA-expressing cells can be visualized under the dorsal ectoderm (data not shown). These are the dorsal cluster neurons. As germ band retraction proceeds, two other clusters of hybndizing cells appear, and all three clusters change shape and grow as the embryo develops (Fig. 1G). This embryo is at about stage $12 / 5$ of Klambt et al. (1991). These authors have subdivided stage 12 into three substages, $12 / 5,12 / 3$, and $12 / 0$, where the second number corresponds to the number of segments still on the dorsal side of the embryo. Fig. 2A shows in situ hybridization to neurons of the ventral and lateral clusters in a stage 14 embryo. Fig. 2B shows the lateral and dorsal clusters. All of the PNS neurons appear to express fasciclin I RNA, as do many of their support cells.

The early cells of the PNS also express fasciclin I protein. This stanning is less obvious than in in situ hybridization experiments, because the surrounding ectoderm also stains with the antibody (Fig. 3C,D; stage $12 / 0$ ). This ectodermal staining could be due to soluble fasciclin I which is bound to these cells (Hortsch 
and Goodman, 1990), as we do not see clear expression of fasciclin I RNA in the ectoderm at this stage by in sttu hybridization.

Later in development fasciclin I protein is expressed on the surfaces of all cell bodies and axons in the PNS (Fig. 3B). The ectodermal expression disappears shortly after germ band retraction is completed. Fasciclin I protein is also observed on the surfaces of glial cells that enwrap the PNS axon bundles (F1g. 3A). In addition to the segmentally repeated PNS cells, there are several specialized sensory structures in the head and tail. Most or all of the cells in these organs also express fasciclin I protein (Zinn et al., 1988). Fasciclin I RNA and protein are retained by some or all of the PNS cells, albeit at reduced levels, until the embryo develops cuticle and it is no longer possible to do whole-mount staining (about $15 \mathrm{~h}$ AEL; Fig. 1H shows in situ hybridization to a stage 16 embryo).

\section{Fasciclin I us initually expressed by all commussural} axons but then localizes to specific bundles

CNS axonogenesis begins in stage $12 / 5$ with the formation of the commissural tracts. The posterior commissure is established first, followed rapidly by the anterior commissure. The commissures are initially fused at the midline (see Fig. 2D), but then become separated by the midline glial cells (Klambt et al., 1991). By stage 15, a complete orthogonal axon scaffold has formed, with anterior and posterior commissures, a bilaterally symmetric pair of longitudinal axon tracts which extend the length of the embryo, and two peripheral nerve roots per hemisegment (the ISN and the segmental nerve (SN)), where motor-neuron axons exit and sensory neurons enter the CNS.

Most or all of the initial commissural axons express fasciclın I protein at approximately equal levels (Figs $2 \mathrm{D}, 4 \mathrm{~A})$. The ventral unpaired midline neurons (VUMs), whose growth cones and axons lie at the junction between the fused anterior and posterior commissures at stage $12 / 0$, express fasciclin I on both their cell bodies and axons at this stage (Fig. 4A). This expression is transient, and disappears shortly after the separation of the commissures. Other early neurons expressing fasciclin I protein on their cell bodies include the aCC and RP1 cells, and a large laterally located cluster of neurons. In contrast to the transient VUM expression, these cells continue to express the protein for several hours. aCC and RP1 are motoneurons and extend axons in the ISN pathway. Fasciclin $I$ is expressed on this pathway at high levels throughout development.

Shortly after the commissures have separated, the expression of fasciclin I on commissural axons becomes restricted to a single large bundle at the posterior edge of the posterior commissure, adjacent to the $\mathrm{aCC}$ cell bodies, and a smaller bundle in the middle of the anterior commissure (Fig. 4D,E). The protein disappears from the rest of the commissural axon bundles. The axons of the lateral cluster neurons continue to express fasciclin I, and most of these axons form a tight bundle which appears to join the fasciclin-I positive
Fig. 1. Timelıne of fasciclin I mRNA and protein expression in the developing Drosophila embryo. In situ hybridization to embryos with fasciclin I cDNA probes was performed using the digoxigenin whole-mount procedure of Tautz and Pfeifle (1989). Fasciclin I protein expression was detected using the $6 \mathrm{D} 8 \mathrm{mAb}$ and HRP immunocytochemıstry. (A) Fasciclin I mRNA localızatıon in a late syncitial blastoderm embryo (stage 4). The arrowhead indicates a nng of higher fasciclin I mRNA concentration The localization of this RNA appears similar to that of the basally localized transcripts described by Davis and Ish-Horowicz (1991). Antenor is to the left. (B) Protein localization in a stage 5 embryo. Anterior is to the right, dorsal up. The large arrowhead on the right indicates the border between low anterior and higher postenor expression of fasciclin I. This border corresponds approximately to the postenor edge of the acron. The two small arrowheads on the left delimit the membrane adjoining the pole cells, which has very high levels of fasciclın I proteın (black staınıng) (C) Protein localızation in a gastrulatıng embryo (stage 6). The embryo is viewed from the ventral side, and anterior is to the left Fasciclin I is expressed on all cell membranes, but at somewhat higher levels on cells at the edges of the ventral furrow (axis marked by smaller arrowhead on the right) and cephalıc furrow (axis marked by larger arrowhead on the left). (D) mRNA localization in a stage 9 germ band extended embryo. 14 stripes of expression are visible (one stripe indicated by arrowhead); these appear to extend through the whole depth of the germ band and therefore include both ectodermal and mesodermal cells Anterior is to the right, dorsal up. (E) Protein localızation in a stage 10 embryo. Segmental stripes or patches of high-level expression are visible (arrowhead). Unlıke the mRNA stripes, however, they appear to be localized to the neuroectoderm layer of the germ band Ectodermal and mesodermal cells express fasciclin I at lower levels Expression is also observed in the cells of the posterior midgut and proctodeum. Anterior is to the left, dorsal up. (F) mRNA localization in a stage 11 embryo. Segmental stripe expression is no longer visible; instead expression is primanly restricted to the neuronal layer, and extends the length of the germ band (large arrowhead) Segmentally repeated thickenings of this longitudinal stripe are observed, however, indicatıng that subsets of neurons express the mRNA at higher levels. The small arrowhead indicates the brain region, whıch also expresses fasciclın I Anterior is to the right, dorsal up. (G) mRNA localization in an embryo undergoing germ band shortening (stage 12). Segmentally repeated clusters of PNS cells are observed. The arrowhead points to a developing dorsal cluster. Antenor is to the left, dorsal up. (H) mRNA localization in a stage 16 embryo, after the beginning of nerve cord condensation The left arrowhead indicates the brain (out of focus). The middle arrowhead points to the dorsal cluster of a PNS cell group It is possible that only a subset of PNS cells express fasciclin I at this stage. The right arrowhead indicates the gonad, which expresses fasciclin I mRNA and protein at high levels. Anterior is to the left, dorsal up.

bundle in the posterior commissure (Fig. 4D,E). There is also a second, smaller fasciclin I-positive tract connectıng these neurons to the anterior commissure. The protein is also expressed on a single bundle at the 

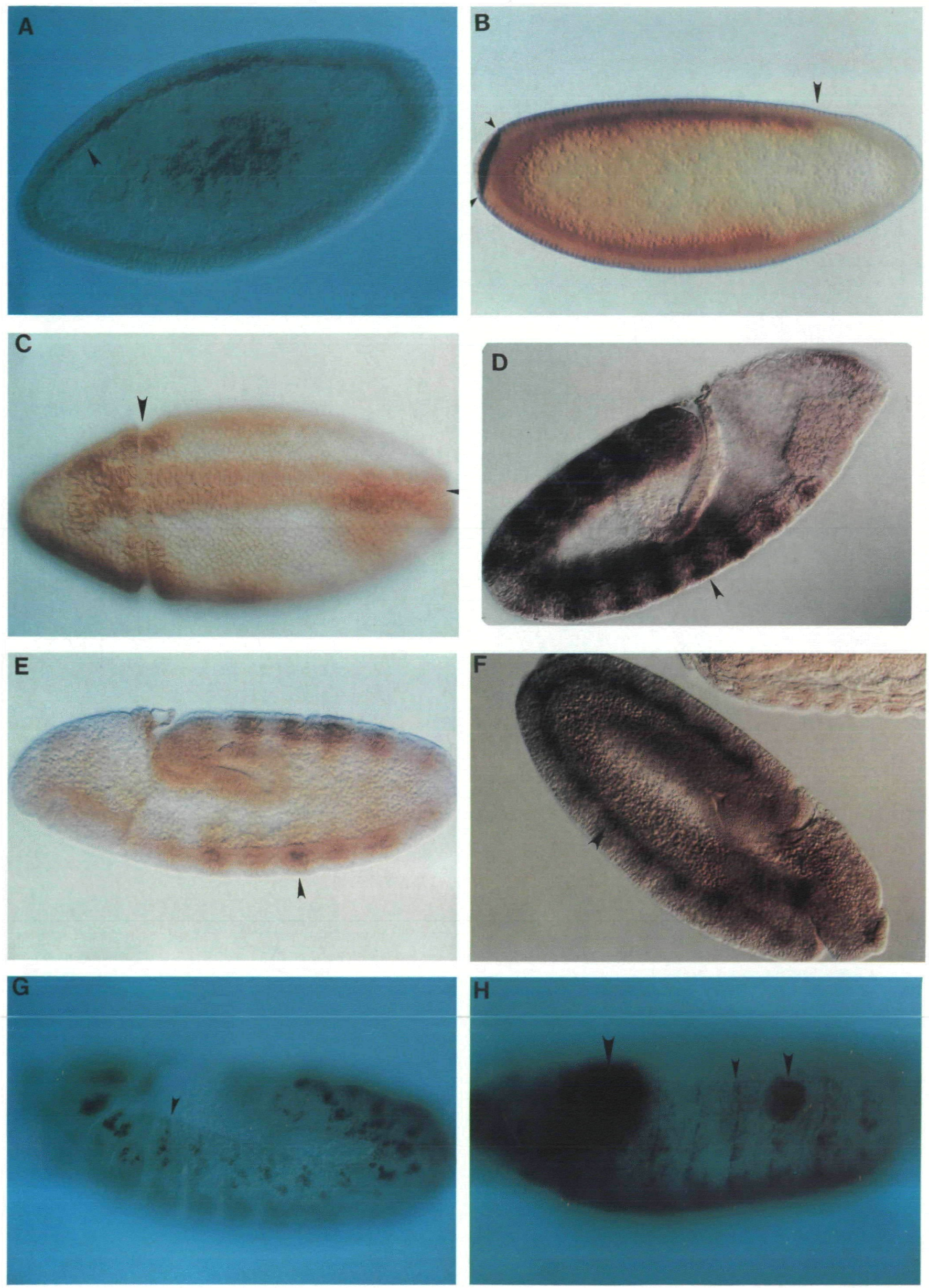
Fig. 2. Fasciclin I mRNA and protein expression in the PNS and CNS. mRNA was detected as in Fig. 1. (A) mRNA localization in the ventral and lateral clusters of the PNS in a stage 14 embryo. The small arrowhead at the top indicates a thoracic lateral cluster, and the larger arrowhead directly below is a larger

abdomınal cluster. The small arrowhead on the left points to a small ventral cluster of neurons, and the large arrowhead on the right to an abdominal dorsal cluster. The dark, out-of-focus staining on the left is the CNS. Anterior is up, dorsal to the right. (B) mRNA localızation in the dorsal and lateral clusters of the PNS in an embryo of a similar age. The large arrowhead at the top indicates a larger thoracic dorsal cluster, and the small arrowhead below it a smaller abdominal dorsal cluster. The large arrowhead on the left points to an abdominal lateral cluster. The cells of the dorsal epidermis, which express fasciclin I mRNA at high levels, form an out-of-focus vertical line at the right, indicated by the righthand arrowhead. Anterior

is up, dorsal to the right. (C) mRNA localızation within the CNS at stage 14. A ventral view, anterior up, is shown. Segmentally repeated clusters of expressing cells are visible ( 7 complete segments). Note the lack of expression along the midline at this stage The large arrowhead indicates one of the CNS lateral clusters of neurons. The cells located medial and slightly posterior to the lateral cluster may include the RP1 neurons; posterior to these cells is another group which may include the aCC and U neurons. The small arrowhead at the left indicates a group of PNS cells The segmental PNS clusters can be seen extending to the right and left of each CNS segment. (D) Fasciclin I protein expression in the CNS of a stage 12/3 embryo. This embryo was dissected and stained with rat anti-fasciclin I antiserum $(1: 200)$ and fluorescein-conjugated goat anti-rat second antibody. Anterior is up. Four complete segments are shown. Note that the commissures, which have developed to slightly different extents in the four segments, are completely fused at this stage (indicated by large arrowhead). The two small vertical arrowheads at the left indicate the developing ISN pathways in two of the segments, these extend toward the periphery. The two small arrowheads at the top point to the migrating RP1 cell bodies, which are moving toward the midline and will eventually lie adjacent to each other (see schematic diagram in Fig. 5). The pattern of axon staining at this stage is similar to that observed with the BP102 mAb, which labels all CNS axons, indicating that most or all of the commissural axons express fasciclin I (see Klambt et al., 1991 for the early BP102 pattern).
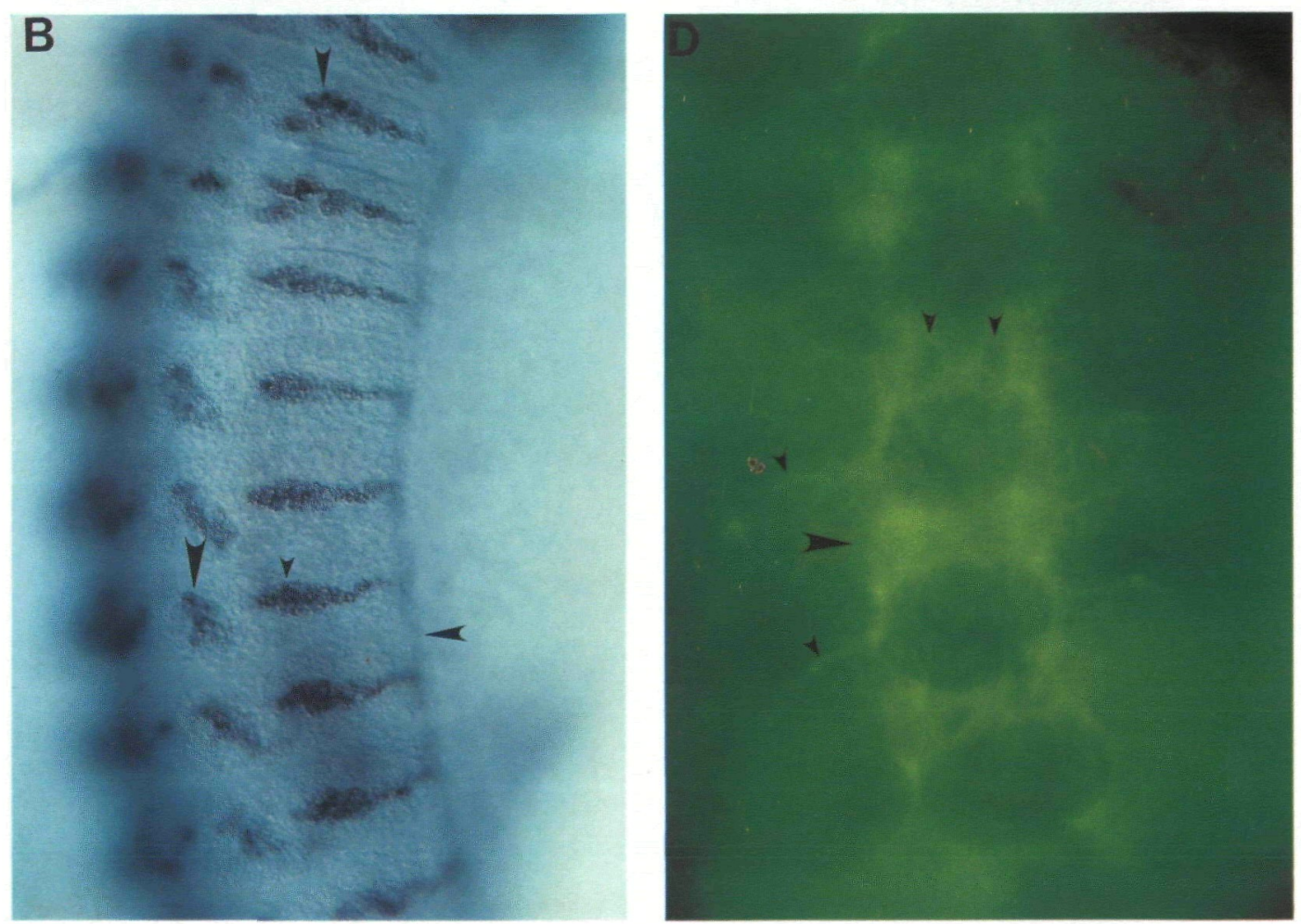
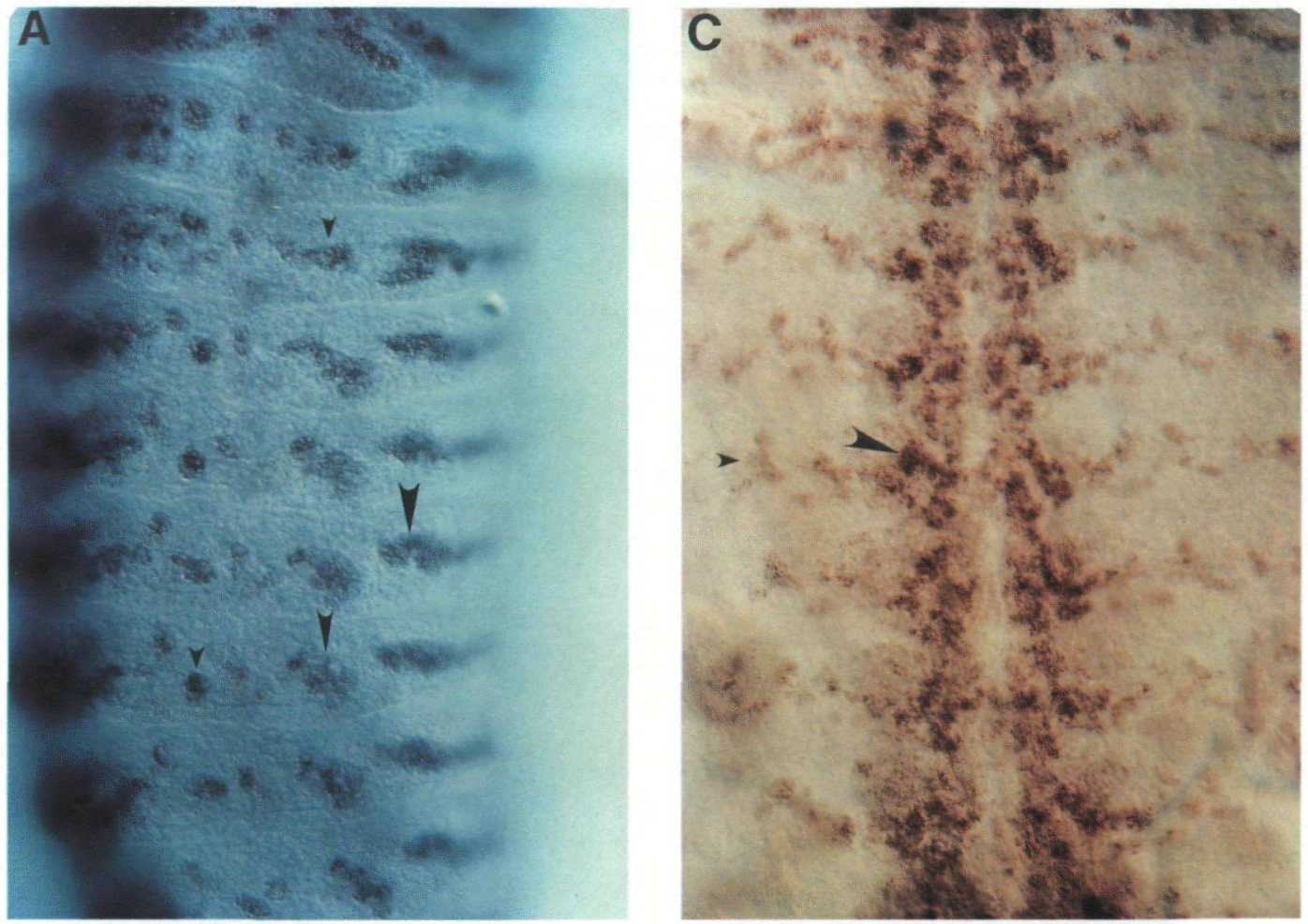

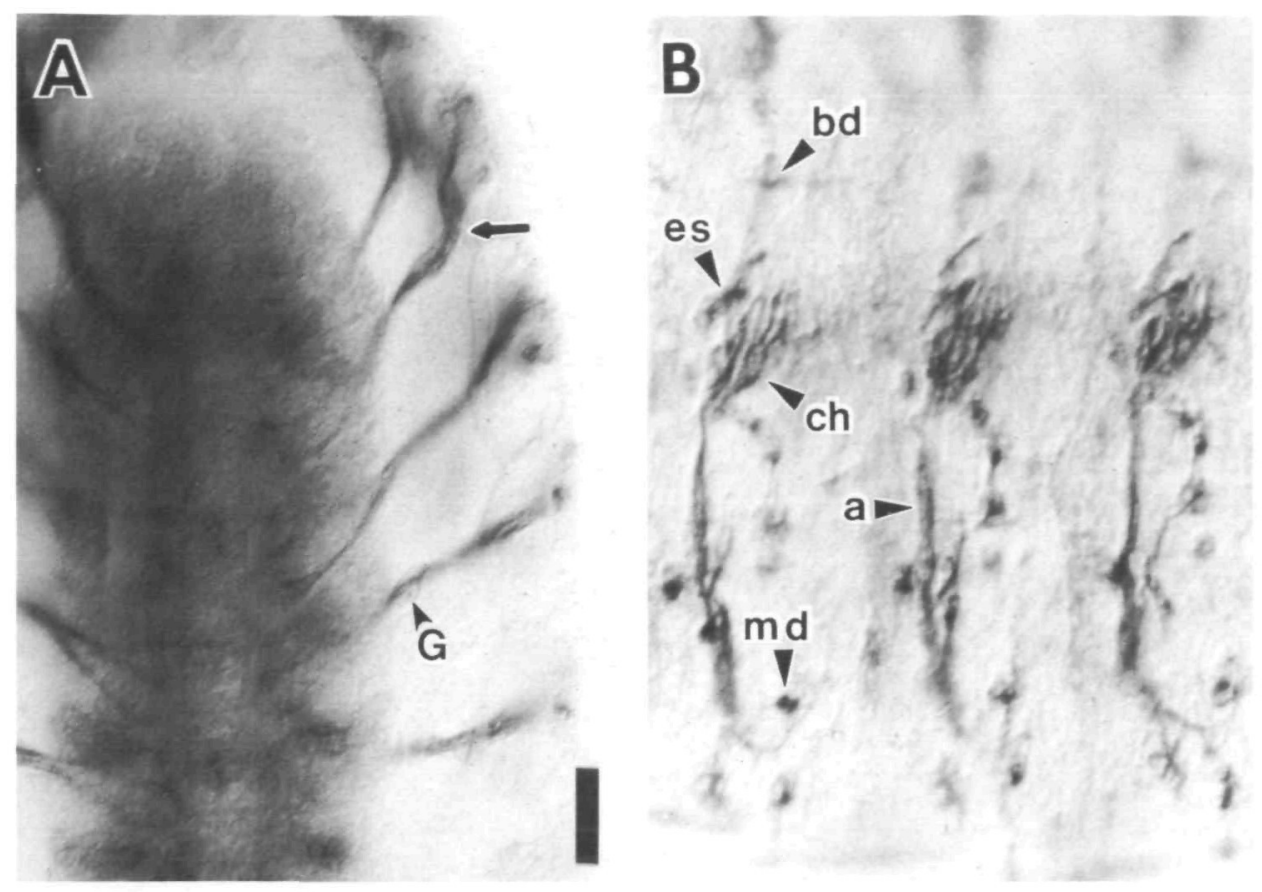

Fig. 3. Expression of fasciclin I protein in the developing PNS. The $6 \mathrm{D} 8 \mathrm{mAb}$ was used for immunocytochemistry. In A, $\mathrm{C}$, and $\mathrm{D}$ anterior is up, and in $\mathrm{B}$ anterior is to the left. (A) A ventral view of the antenor end of a late stage $15 \mathrm{AEL}$ embryo The segmental and intersegmental nerves are seen extending out to the periphery (arrow). They come together at the edge of the CNS, and at their junction a fasciclin I positive glial (G) cell is seen (B) The mature embryonic PNS of three abdominal segments in an earlıer stage 15 embryo Most or all of the cellular constituents express fasciclın I. The lateral cluster with chordotonal (ch) and external sensory (es) organs and the ventral clusters with several strongly staining multiple dendrite cells (md) are in the focal plane. Also visible is the bipolar dendrite (bd) cell of the lateral cluster which expresses fasciclin I, as does the support cell associated with this neuron (not shown here). (C) Expression in the PNS of stage 12 embryo. Fasciclin I proten is expressed on both early PNS and ectodermal cells, but the PNS neurons have higher levels of expression. The arrowhead indicates an abdomınal dorsal cluster of neurons. $g$, the developing gonad (out of focus). (D) An embryo of a simular age to the one in $\mathrm{C}$; abdominal lateral clusters are shown with chordotonal neurons (ch) and their support cells, called cap cells (C), indicated. Bar, $25 \mu \mathrm{m}$ (A); $12.5 \mu \mathrm{m}$ (B-D).

medial edge of the longitudinal tract. The changing pattern of fasciclin I protein expression in the CNS is schematically depicted in Fig. 5.

In situ hybridization shows that fasciclin I RNA is always restricted to a subset of CNS neurons (Fig. 2C), and there is no evidence that this subset is larger at early stages of CNS development. Thus, the early expression of fasciclin I protein on all commissural axons may indicate that a subset of neurons that express fasciclin I RNA (perhaps at low levels) pioneer the commissures, or that fasciclin I cleaved by an endogenous phospholi- pase (Hortsch and Goodman, 1990) can bind to axons of nonexpressing cells.

\section{Expression of fasciclin I in nonneural tissues}

In addition to the early nonneural expression described above, fasciclin I RNA and protein are also observed on a variety of other cells and tissues during development. The cells at the dorsal edge of the epidermis, which grow together over the amnioserosa during the process of dorsal closure, express fasciclin I RNA and protein at high levels (Fig. 2B). This process is completed during 
Fig. 4. Expression of fasciclin I protein in the developing CNS. The $6 \mathrm{D} 8 \mathrm{mAb}$ was used for immunocytochemistry. Embryos were cleared in methyl salicylate and nerve cords were 'cracked out' under cover slips. Anterior is up for all nerve cords. (A) shows 2.5 segments of the dorsal surface of a nerve cord of a stage $12 / 0$ embryo, somewhat older than the embryo in Fig. 2D). The anterior and posterior commissures (the left arrowheads) are still attached by the VUM axons (the right arrowhead). Both the commissural axons and the VUM axons express fasciclin I. Few of the longitudinal axons have extended between the segments at this time Some fasciclin I-positive lateral cells are seen near the left arrowheads Four segments of a stage 13 embryo are shown in (C). The fasciclin I-positive commissures (right arrowheads) have separated and the VUMs and their axons no longer express the protein Cells of the lateral cluster (left arrowhead) express fasciclin I. One and one-half segments of a slightly older nerve cord are shown in (B). The

commissural expression has decreased and the fasciclin I-positive RP1 cell body (left arrowhead) and aCC cell bodies (lower arrowheads) can be identified very near to their characteristic positions RP1 is eventually localized between the commissures, and aCC just posterior to the posterior commissure. A single bundle in the postenor commissure has begun to express slightly higher levels of fasciclin I than do the other bundles. As

shown in (D) and (E), the axonal expression of fasciclin I protein resolves to a single bundle (left arrowheads) in the posterior comissure of the later embryonic nervous system (stage 15, five segments shown). There is also a smaller fasciclın I-positive bundle in the anterior commissure; this bundle is out of the plane of focus The embryo in E is slightly older than that in D, and the difference in expression between the fasciclin I-positive postenor commissure bundle and the other bundles is more pronounced. The fasciclin I-positive lateral cluster of cells (nght arrowheads) extends a tight bundle of axons, which also express the protein, into the posterior commissure. Bar, $18 \mu \mathrm{m}(\mathrm{A}) ; 14 \mu \mathrm{m}$ (B-E).

stage 15 , and shortly after dorsal closure these cells cease to express fasciclin I.

The gonads express fasciclin I RNA (Fig. 1H) and protein (data not shown), and this expression continues until cuticle has formed and the embryo is no longer accessible. We do not know precisely when this expression begins, as it is difficult to visualize the pole
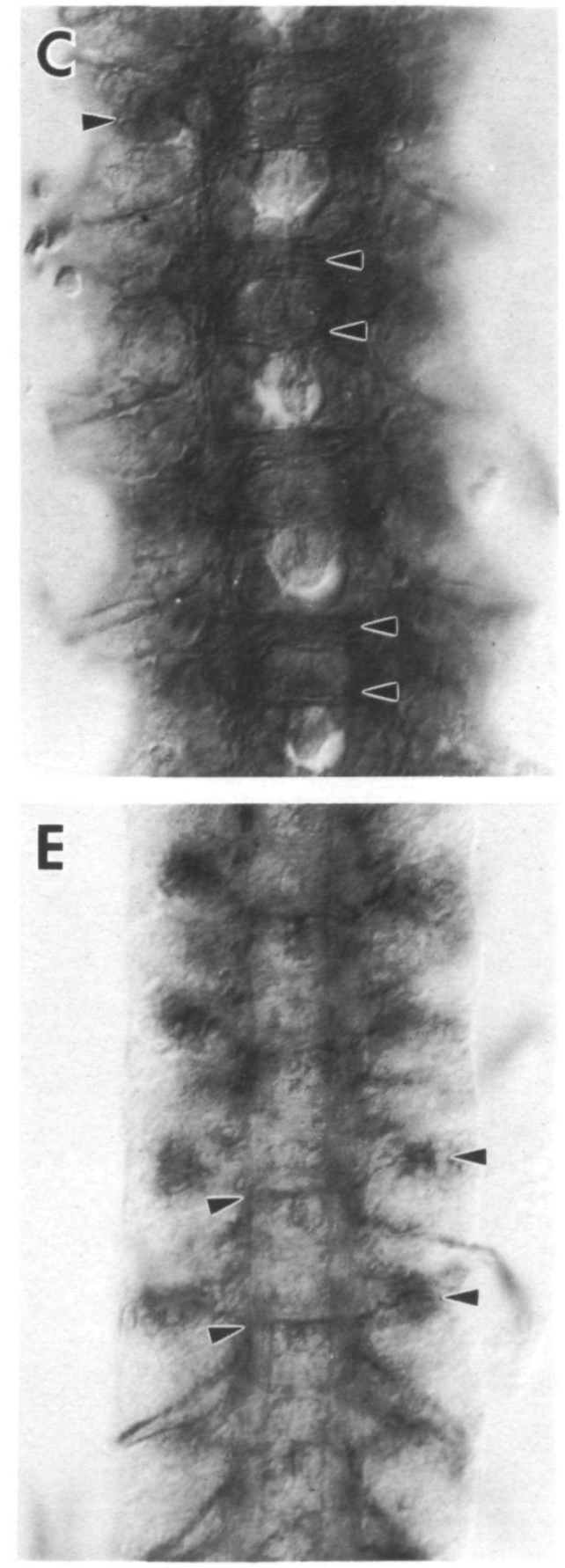


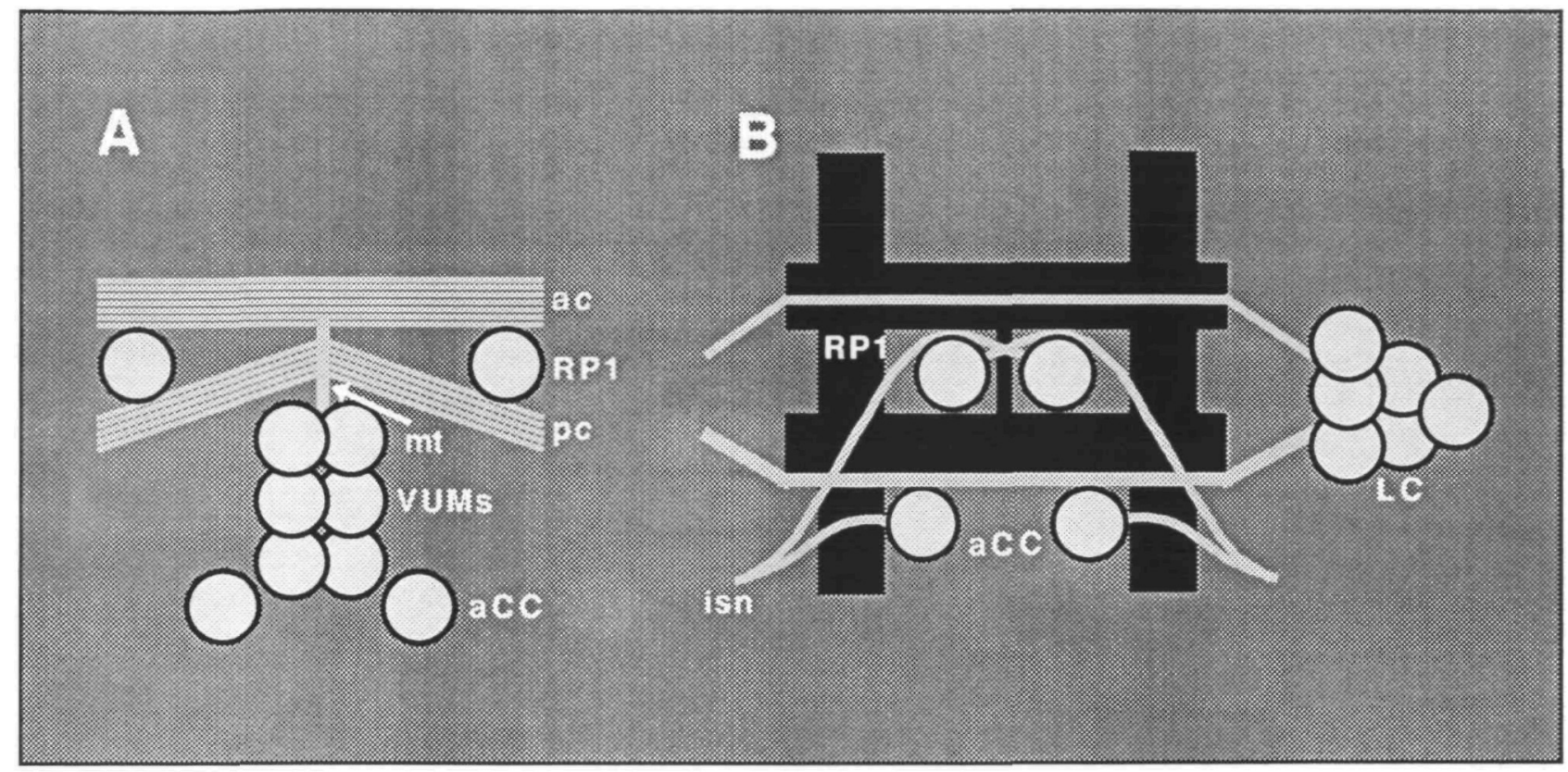

Fig. 5. Schematic diagram of the dynamic expression of fasciclin I protein in the developing CNS. The diagrams in (A) and (B) show a single segment of the Drosophila CNS. The thin gray bars indicate fasciclin I-positive axon bundles. All the cell bodies shown (gray circles) are fasciclin I-positıve; other cell bodies are not depicted. The segment in (A) is typical of a stage $12 / 2$ embryo, in which germ band retraction is not entirely completed The commissures have formed (light bars with fine lines) but there are very few longitudinal axons. The commissures are still fused at the midline. Fasciclin $I$ is expressed on the VUM cell bodies (VUMs) positioned at the dorsal midline and on their axons, which form the medial tract (mt) and then bifurcate in the commissures. It is also expressed on most if not all of the early axons in both commissures (ac,pc). The fasciclin I-positıve RP1 cell bodies, initially located laterally, migrate toward the midlıne whıle the commissures are still fused. They eventually insert themselves between the two commissures. The aCC cell bodies, enroute on their anterior migration from the next posterior segment, also express fasciclin I. (B) A segment of the CNS in a stage 15 embryo The commissures have separated, and the VUMs and their axons no longer express fasciclin I. The orthogonal CNS axon scaffold is indicated by the wide black bars, and the fasciclin I-positive bundles by the thin gray bars. The RP1 cells are situated between the commissures near the midline and both the cell bodies and their axons are fasciclın Ipositive The aCCs lie just posterior to the posterior commissure; their soma and axons also express fasciclin I. The aCC and RP1 axons extend together out the intersegmental nerve (isn). A single bundle in each of the commissures now expresses fasciclin I. Many of the axons in these fasciclin I-positive bundles are likely to originate in the lateral cluster (LC) situated outside the longitudinal connectives and between the commissures. Only one of the two bilaterally symmetric clusters is shown here. These LC neurons express fasciclin I beginning at stage $12 \mathrm{AEL}$ and continue to do so until at least stage 16.

the salivary glands. Sections of the posterior midgut and of the hindgut also express the protein. Finally, fasciclin I protein expression is observed on the anterior and posterior spiracles, which are the outlets of the tracheae. Both neural and nonneural expression of fasciclin I protein is eliminated by the fas $I^{T E}$ mutation, showing that all of the $\mathrm{mAb}$ staining observed is due to authentic fasciclin I (data not shown).

\section{Discussion}

Dynamic expression of neural adhesion molecules during embryogenesis in Drosophila

The results described above show that the cell adhesion molecule fasciclin I is expressed in a complex pattern throughout embryonic development. The protein is uniformly expressed on nascent cell membranes at the end of the synctitial blastoderm stage. It is briefly distributed in a graded pattern in the cellular blastoderm (Fig. 1B), after which expression again becomes uniform. In the gastrulating embryo all cells express fasciclin I, but invaginatıng cells have higher levels of the protein on their surfaces (Fig. 1C).

During the germ band extended stage, fasciclin I expression evolves through two distinct phases. In stage 9-10 embryos, fasciclin I is localized to 14 segmentally repeated stripes of neuroepithelial cells and neuroblasts (Fig. 1D, E). During stage 11, fasciclin I RNA and protein disappear from the neuroblast layer and become concentrated in a single continuous longitudinal stripe of CNS neurons (Fig. 1F). The early cells of the PNS, which first become visible just prior to germ band retraction, also express fasciclin I RNA (Fig. 1G) and protein (Fig. 3C, D). As germ band retraction proceeds, fasciclin I protein expression on the ectoderm increases, and later disappears during stage 14 (Fig. 3; compare $\mathrm{B}$ with $\mathrm{C}$ and D). Expression on the cell bodies and axons of the PNS, in contrast, is stable; the surfaces of these cells retain fasciclin I protein until the embryo is no longer accessible to antibody. 
The CNS expression pattern of fasciclin I during axonogenesis can be described as a combination of transient and stable components. During the formation of the commissures (stage 12), fasciclin I protein is found on most or all axons (Figs 2D, 4A-C). This pattern of expression lasts for less than an hour. After this time, fasciclin I is expressed at high levels only on a single bundle in each commissure, on a bundle in the longitudinal tract, and on the axons of the ISN and SN (Fig. 4D,E). The cell bodies of the VUM neurons express fasciclin I during the commissural formation phase, but become fasciclin-I negative thereafter. In contrast, the aCC, RP1, and lateral cluster neurons maintain fasciclin I expression on their cell bodies until at least stage 16 (summarized in Fig. 5).

In addition to neural expression, the germ band retracted embryo expresses fasciclin I in a variety of nonneural tissues. These include the dorsal edge of the epidermis, the salivary glands, the gonads, sections of the hindgut and posterior midgut, and the spiracles. The duration of fasciclin I expression varies between these different tissues. For instance, the dorsal epidermis expresses fasciclin I only during the process of dorsal closure. In contrast, expression in the gonads is very stable.

The expression patterns of the two other Drosophila fasciclins have many of the characteristics of the fasciclin I pattern described here. Fasciclins II and III are both dynamically expressed in neural and nonneural tissues. In the CNS, fasciclin III is transiently expressed on 5 commissural bundles and on part of the ISN pathway. Like fasciclin $I$, it also appears on a segmentally repeated subset of neuroepithelial cells and underlying neuronal lineages at the germ band extended stage. At this stage, it is also expressed on patches of epithelial cells near the stomodeal and proctodeal invaginations and on the visceral mesoderm. After germ band retraction, it is expressed on luminal cells of the salivary gland, and in a 14-stripe pattern on the ectoderm. These stripes coincide with the segmental grooves (Patel et al., 1987). Fasciclin II is initially expressed on a single longitudinal CNS pathway, and appears on several other pathways later in development. It is also expressed in patches of ectoderm from which the spiracles arise, as well as in a section of hindgut and in the malpighian tubules (Grenningloh et al., 1991).

These results suggest that the function of neural adhesion molecules in Drosophila embryogenesis is not restricted to nervous system development. These molecules are likely to be used for morphogenesis and adhesion in a variety of different tissues. In some cases they may form stable adhesive zones that are involved in maintaining the structure of a particular organ. In tissues where they are only transiently expressed, they may facilitate morphogenetic movements or cell signaling events.

Comparison of fasciclin I expression patterns in grasshopper and Drosophila

Although grasshoppers and Drosophila develop to adulthood in very different ways, the overall structure of the early embryonic CNS is very similar in the two organisms. This resemblance extends to the axon trajectories of certain identified neurons (Thomas et al., 1984). Comparison of the expression patterns of fasciclin I in the two species might thus yield insights into its function.

In both grasshopper and Drosophila, fasciclin I is expressed at an early stage on segmentally repeated subsets of neuroepithelial cells. Later in development, all PNS cell bodies and axons express fasciclin I in both insects. In the grasshopper CNS, fasciclin I protein is localized on a single bundle in each commissure, on a bundle in the longitudinal tract, and on the axons of the ISN. This pattern is almost identical to the later expression in the Drosophula CNS, and this resemblance extends to particular identified cells such as aCC and RP1, which express the molecule in both organisms (Bastiani et al., 1987; Zinn et al., 1988; this work).

The pattern of fasciclin I expression in grasshopper and Drosophlla differs in two major ways. First, fasciclin $I$ is localized to patches of epithelium in the grasshopper limb buds. There are no counterparts to these structures in fly embryos. Secondly, the early, transient phase of CNS expression in Drosophila is not observed in grasshopper. In Drosophila, the molecule is initially expressed on all commissural axons and later becomes restricted to particular bundles. In contrast, fasciclin I is localized to specific pathways from the beginning of axonogenesis in grasshopper. This may correlate with differences in the way the commissures are established in the two insects. In Drosophila, the two commissures are initially fused and then become separated by insertion of a midline glial cell, MGM (Klambt et al., 1991). In the much larger grasshopper embryo, the anterior and posterior commissures are always separate.

\section{Fasciclin I CNS expression and the phenotype of fasI/abl mutants}

The fasI/abl phenotype (absence of commissural axon tracts) is clearly not due to a failure of pathfinding by those axons that comprise the fasciclin I-positive bundles observed in later Drosophila embryos (Fig. 5), because all commissural axons are affected. Rather, it appears that there is a general defect in growth cone extension across the midline (Klambt et al., 1991). This correlates well with the early, transient expression of fasciclin I on all commissural axons reported here.

We do not know what mechanism accounts for the failure of commissural axons to cross the midline in fasl/abl embryos. The specialized midline glia and neurons are essential for commissure formation, but these seem to be in their normal positions in mutant embryos (Elkins et al., 1990a). One possibility is that commissural growth cones must recognize and interact with the VUM cells, whose growth cones and nascent axons lie at the fusion point of the two commissures. In orthodenticle mutants the VUM cells die and the posterior commissure does not form (Klambt et al., 1991). Since fasciclin I has been shown to be a 
homophilic adhesion molecule in tissue culture (Elkins et al., 1990b), and the commissural axons and VUM cells both express the molecule at this stage, it is possible that elimination of fasciclin I-mediated growth cone adhesion to the VUMs is a component of the fasl/abl phenotype.

Because the commissures are absent in fasI/abl embryos, we cannot determine whether fasciclin I also has a role in specific pathfinding by later growth cones that might use the fasciclin I-positive bundles as guidance cues. This is analogous to the situation in slit mutant embryos, where the early nervous system collapse phenotype (due to the absence of midline slit expression) prevents the observation of a possible phenotype correlated with the later expression of slit product on CNS axons (Rothberg et al., 1990).

FasI/abl embryos are essentially normal except for their alterations in the CNS axon array (Elkins et al., 1990a). This is probably because the CNS is the major tissue in which both fasciclın I and $a b l$ are expressed. The double mutant phenotype would thus be most likely to be observed in the CNS. Fasciclin I expression outside the CNS is likely to have a functional role, however, since its pattern has been conserved over the 300 million years of evolution that separate Drosophla from grasshoppers. In order to uncover such a role, it will be necessary to find mutations in other genes that produce a synergistic phenotype when combined with fasI mutations.

A possible developmental role that is consistent with the early nonneural expression of these apparently redundant adhesion molecules could be to ensure that morphogenetic movements take place normally in all embryos. We have observed that in collections of 11$13 \mathrm{~h}$ old embryos from wild-type stocks, about $1 \%$ of embryos are severely abnormal. The most common phenotype observed in these embryos is a failure of germ band retraction; gastrulation/germ band extension defects are also observed. In egg collections from homozygous fasI flies, the percentage of these abnormal embryos increases to $10-12 \%$, and, in collections from homozygous fasI/fasIII stocks, to $30-35 \%$. This phenotype of fasI mutants can be rescued by P-element transformants containing the fasciclin I gene (K. Zinn, T. Elkins, and C. S. Goodman, unpublished results).

This result suggests that these and other cell adhesion molecules could act as 'correctors' in morphogenesis. We hypothesize that although most mutant embryos lacking a particular adhesion molecule will be able to develop normally, embryos in which cells are in abnormal positions may fail to develop because they are unable to correct these defects. The fraction of embryos in this class would increase still further if multiple correcting functions are removed by mutation, because the extent of tolerable abnormality in cell position would be decreased as each molecule was eliminated (the 'window of normality' would shrink in size). That such a correcting mechanism actually exists is illustrated by embryos derived from mothers having different numbers of copies (1, 3 or 4 ) of the bicold gene, which determines anterior cell fates. These embryos vary greatly from normal in the positions of their cephalic furrows and in the placement of parr-rule gene expression stripes, yet they all develop into normal larvae and adults (Driever and Nusslein-Vollhard, 1988).

Involvement in a correcting mechanism could provide a selection for the evolution of genes encoding new cell adhesion molecules, even if they do not initially have functions independent of those of previously existing molecules. The new adhesion proteins could then evolve new roles and patterns of expression that would allow the development of greater complexity in the nervous system.

We thank Shin-Shay Tian for performing some of the whole-mount in situ hybridization experiments, Michael Hortsch for the anti-fasciclın I mAbs and for help with initial immunocytochemistry experiments using these mAbs, Karen Jepson-Innes and Susan Parkhurst for help with the whole mount in situ technique, Dolores Ferres-Marco for help with ımmunocytochemistry, Yash Hıromı for a stock carrying the $f t z$-lac Z insertion on the X chromosome, Bruce Alberts for helpful discussions concernıng bicold phenotypes, and Nipam Patel for invaluable advice This work was supported by grants from the NIH to C S.G. and K.Z.

\section{References}

Bastiani, M. J., Harrelson, A. L., Snow, P. M. and Goodman, C. S. (1987) Expression of fasciclin I and II glycoproteins on subsets of axon pathways during neuronal development in the grasshopper Cell 48, 745-755.

Bodmer, R., Carretto, R. and Jan, Y. N. (1989) Neurogenesis of the peripheral nervous system in Drosophlla embryos. DNA replication patterns and cell lineages Neuron 3, 21-32

Campos-Ortega, J. A. and Hartenstein, V. (1985) The Embryonic Development of Drosophla melanogaster Berlın Springer-Verlag

Chang, S., Rathjen, F. G. and Raper, J. A. (1987) Extension of neurites on axons is impaired by antibodies against specific neural cell surface glycoproteins $J$ Cell Biol 104, 355-362

Davis, I. and Ish-Horowicz, D. (1991) Apical localuzation of pair-rule transcnpts requires $3^{\prime}$ sequences and limits protein diffusion in the Drosophla blastoderm embryo. Cell 67, 927-940

Driever, W. and Nusslein-Vollhard, C. (1988). The bicoud protein determines position in the Drosophila embryo in a concentrationdependent manner Cell 54, 95-104

Elkins, T., Hortsch, M., Bleber, A. J., Snow, P. M. and Goodman, C. S. (1990b) Drosophila fasciclin I is a novel homophilic adhesion molecule that along with fasciclin III can mediate cell sorting. $J$ Cell Biol 110, 1825-1832

Elkins, T., Zinn, K., McAllister, L., Hofimann, F. M. and Goodman, C. S. (1990a). Genetic analysis of a Drosophula neural cell adhesion molecule interaction of fasctclin $I$ and Abelson tyrosine kinase mutations Cell 60, 565-575

Gertler, F. B., Bennett, R. L., Clark, M. J. and Hoffman, F. M. (1989) Drosophila abl tyrosine kınase in embryonic CNS axons' a role in axonogenesis is revealed through dosage-sensitive interactions with disabled Cell 58, 103-113

Ghysen, A., Dambly-Chaudiere, C., Aceves, E., Jan, L. Y. and Jan, Y. N. (1986). Sensory neurons and penpheral pathways in Drosophula embryos Roux. Arch Devl Bıol 1-9

Goodman, C. S., Bastiani, M. J., Doe, C. Q., du Lac, S., Helfand, S. L., Kuwada, J. Y. and Thomas, J. B. (1984). Cell recognition during neuronal development Sclence 225, 1271-1279

Grenningloh, G., Bieber, A. J., Rehm, E. J., Snow, P. M., Traquina, Z., Hortsch, M., Patel, N. H. and Goodman, C. S. (1990) Molecular genetics of neuronal recognution in Drosophila evolution and function of immunoglobulin superfamily cell adhesion molecules Cold Spring Harbor Symp Quant Biol 55, $327-340$ 
Grenningloh, G., Rehm, E. J. and Goodman, C. S. (1991) Genetic analysis of growth cone guidance in Drosophila fasciclin II functions as a neuronal recognition molecule Cell 67, 45-57

Hafen, E., Kuroiwa, A. and Gehring, W. J. (1984) Spatial distribution of transcripts from the segmentation gene fiz durng Drosophila embryonic development. Cell 37, 833-841

Harrelson, A. L. and Goodman, C. S. (1988) Growth cone guidance in insects: fasciclin II is a member of the immunoglobulin superfamily Science 242, 700-708

Henkemeyer, M. J., Gertler, F. B., Goodman, W. and Hoffman, F. M. (1987) The Drosophila Abelson proto-oncogene homolog identification of mutant alleles that have pleiotropic effects late in development Cell 51, 821-828

Hortsch, M. and Goodman, C. S. (1990) Drosophula fasciclin I, a neural cell adhesion molecule, has a phosphatıdylınositol lipid membrane anchor that is developmentally regulated $J$ bol Chem 265, $15104-15109$

Klambt, C., Jacobs, J. R. and Goodman, C. S. (1991). The midlıne of the Drosophila central nervous system. a model for the genetic analysıs of cell fate, cell migration and growth cone guidance Cell 64, 801-816

McAllister, L., Rehm, E. J., Goodman, C. S. and Zinn, K. (1992) Alternative splicing of micro-exons generates multıple forms of the insect cell adhesion molecule fasciclin I. $J$ Neuroscl, in press

Martinez-Arias, A. and Lawrence, P. A. (1985) Parasegments and compartments in the Drosophta embryo Nature 313, 639-642

Neugebauer, K. M., Tomaselli, K. J., Lilien, J. and Reichardt, L. F. (1988) N-cadherin, NCAM, and integrins promote retinal neunte outgrowth on astrocytes in vuro $J$ Cell Biol 107, 1177-1187

Patel, N. H., Martin-Blanco, E., Coleman, K. G., Poole, S. J., Ellis, M. C., Kornberg, T. B. and Goodman, C. S. (1989) Expression of engrauled proteins in arthropods, annelıds and chordates Cell $\mathbf{5 8}$, 955-968.
Patel, N. H., Snow, P. M. and Goodman, C. S. (1987) Characterization and cloning of fasciclin III a glycoprotein expressed on a subset of neurons and axon pathways in Drosophila Cell 48, 975-988

Rothberg, J. M., Jacobs, J. R., Goodman, C. S. and ArtavanisTsakonas, S. (1990) slit an extracellular protein necessary for development of midline glia and commissural axons contains both EGF and LRR domains Genes Dev 4, 2169-2187

Snow, P. M., Bieber, A. J. and Goodman, C. S. (1989) Fasciclin III a novel homophilıc adhesıon molecule in Drosophila Cell 59, 313323

Snow, P. M., Zinn, K., Harrelson, A. L., McAllister, L., Schilling, J. Bastiani, M. J., Makk, G. and Goodman, C. S. (1988) Charactenzation and cloning of fasciclin I and fasciclin II glycoproteins in the grasshopper Proc natn Acad Scl USA 85, $5291-5295$

Tautz, D. and Pfeifle, C. (1989) A nonradioactive in situ hybridization method for the localization of specific mRNAs in Drosophila embryos reveals a translational control of segmentation gene hunchback Chromosoma 98, 81-85.

Thomas, J. B., Bastiani, M. J., Bate, C. M. and Goodman, C. S (1984) From grasshopper to Drosophila a common plan for neuronal development Nature 310, 203-207

Tomaselli, K. J., Reichardt, L. F. and Blxby, J. L. (1986) Distinct molecular interactions mediate neuronal process outgrowth on non-neuronal cell surfaces and extracellular matnces $J$ Cell Bıol 103, 2659-2672.

Zinn, K., McAllister, L. and Goodman, C. S. (1988) Sequence analysis and neuronal expression of fasciclin I in grasshopper and Drosophila Cell 53, 577-587 